\title{
Distinguishing Newly Born Strange Stars from Neutron Stars with $g$-Mode Oscillations
}

\author{
Wei-jie $\mathrm{Fu}^{a}$, Hai-qing $\mathrm{Wei}^{b}$, and $\mathrm{Yu}$-xin $\mathrm{Liu}^{a, c, *}$ \\ ${ }^{a}$ Department of Physics and State Key Laboratory of Nuclear Physics and Technology, \\ Peking University, Beijing 100871, China \\ ${ }^{b}$ oLambda, Inc., Sunnyvale, CA 94089, USA \\ ${ }^{c}$ Center of Theoretical Nuclear Physics, National Laboratory of Heavy Ion Accelerator, \\ Lanzhou 730000, China
}

October 31, 2018

\begin{abstract}
The gravity-mode ( $g$-mode) eigenfrequencies of newly born strange quark stars (SQSs) and neutron stars (NSs) are studied. It is found that the eigenfrequencies in SQSs are much lower than those in NSs by almost one order of magnitude, since the components of a SQS are all extremely relativistic particles while nucleons in a NS are non-relativistic. We therefore propose that newly born SQSs can be distinguished from the NSs by detecting the eigenfrequencies of the $g$-mode pulsations of supernovae cores through gravitational radiation by LIGO-class detectors.
\end{abstract}

PACS Nos. 97.60.Gb 26.60.-c 97.10.Sj 95.85.Sz

\footnotetext{
*Corresponding author, e-mail address: yxliu@pku.edu.cn
} 
Since their discovery forty years ago, pulsars have been serving as unprecedented astrolaboratories, especially due to their extreme density and magnetic field. However, the basic nature of pulsar-like stars remains murky, since, besides the conventional neutron stars (NSs), strange quark stars (SQSs) are also potential candidates for the stars [1, 2, 3, 4, 5, 6, 7], due to the quark deconfinement phase transition at high density. More recently, the composition of pulsars, especially the existence of SQSs, has become a hotly debated topic (see, for example, Refs. [6, 7, 8, 9, 10]), since the observation of the object PSR J0751+1807 yielded a mass $(2.1 \pm 0.2) \mathrm{M}_{\odot}$ with $1 \sigma$ error bars [11], and one of the pulsars Ter $5 \mathrm{I}$ and $\mathrm{J}$ has a mass larger than $1.68 M_{\odot}$ to $95 \%$ confidence [12]. However, in general view, the equation of state (EOS) of quark matter is much softer, the quark star may not be so massive [8]. The composition of pulsars and the method to distinguish the SQSs from NSs becomes a fundamental topic not only in astrophysics but also in high energy physics and nuclear physics.

Many potentially observable differences have been proposed to distinguish SQSs from NSs. For example, SQSs have much larger dissipation rate of radial vibrations [13] and higher bulk viscosity [14]; the spin rate of SQSs can be much closer to the Kepler limit than that of NSs [15]; SQSs may cool more rapidly than NSs within the first 30 years [16]. In this letter, we propose that the eigenfrequencies of the gravity-mode ( $g$-mode) pulsations of supernova cores provide another sharp criteria to distinguish SQSs from NSs after their birth in a core-collapse supernovae.

Recent simulations of core-collapse supernovae have indicated that core $g$-mode pulsations may be excited by turbulence and downstream accretion [17], due to the stalled supernova shock undergoing a standing-accretion-shock instability and becoming highly deformed [18, 19]. Following this discovery, Ott et al. have found that these $g$-mode pulsations of supernova cores serve as efficient sources of gravitational waves [20]. It is natural to expect that more information about the interior constituents of supernova cores carried by gravitational waves, such as whether SQSs can be formed during the processes of supernovae, could be obtained by groundbased detectors such as LIGO and VIRGO. In this letter we present our calculations of the $g$-mode eigenfrequencies for newly born NSs and SQSs in the supernova cores. Our results show that the frequencies of newly born SQSs are lower than those of NSs by as much as one order of magnitude. Such a large difference may provide an appropriate means to distinguish SQSs from NSs that are newly formed in a core-collapse supernovae. The present work is then of general interest in nuclear/quark physics, gravitation-wave physics and supernova physics.

Our calculations start with the differential equations in the Newtonian formulation which governs the linear oscillations of a nonrotating, unmagnetized, and fluidic star (for details, see for example, Refs. [21, 22, 23]). If the equilibrium configuration of a star is assumed to be spherically symmetric, the pressure $p_{0}$, the density $\rho_{0}$, and the gravitational potential $\phi_{0}$ depend only on the radial coordinate $r$. Oscillations of the star can be described by a vec- 
tor field $\boldsymbol{\xi}(\boldsymbol{r}, t)$ of displacement and the Eulerian (or "local") perturbations of the pressure, density, and the gravitational potential are: $\delta p, \delta \rho$, and $\delta \phi$. We adopt the Cowling approximation, i.e., neglecting $\delta \phi[24]$. Let the radial component of $\boldsymbol{\xi}(\boldsymbol{r}, t)$ be $\xi_{r} Y_{l m}(\theta, \varphi) \mathrm{e}^{-\mathrm{i} \omega t}$, following the equations of motion and continuity we have the linear oscillation equations as

$$
\begin{aligned}
\frac{d}{d r}\left(r^{2} \xi_{r}\right) & =\frac{g}{c_{s}^{2}}\left(r^{2} \xi_{r}\right)+\left[\frac{l(l+1)}{\omega^{2}}-\frac{r^{2}}{c_{s}^{2}}\right]\left(\frac{\delta p}{\rho_{0}}\right), \\
\frac{d}{d r}\left(\frac{\delta p}{\rho_{0}}\right) & =\frac{\omega^{2}-\omega_{B V}^{2}}{r^{2}}\left(r^{2} \xi_{r}\right)+\frac{\omega_{B V}^{2}}{g}\left(\frac{\delta p}{\rho_{0}}\right)
\end{aligned}
$$

where the dependence of $\delta p$ on the spherical harmonics $Y_{l m}(\theta, \varphi)$ and the time factor $\mathrm{e}^{-\mathrm{i} \omega t}$ is separated analogous to $\xi_{r} ; \omega$ is the eigenfrequency of a mode with the index $\alpha \equiv\{n, l, m\}$ being abbreviated; $g$ is the local gravitational acceleration, and the Brunt-Väisälä frequency $\omega_{B V}$ is defined as

$$
\omega_{B V}^{2}=g^{2}\left(\frac{1}{c_{e}^{2}}-\frac{1}{c_{s}^{2}}\right),
$$

with $c_{s}, c_{e}$ being, respectively, the adiabatic, the equilibrium sound speeds defined as

$$
c_{s}^{2} \equiv\left(\frac{\partial p}{\partial \rho}\right)_{\text {adia }}, \quad c_{e}^{2} \equiv\left(\frac{d p}{d \rho}\right)_{\text {equi }}=\frac{d p_{0} / d r}{d \rho_{0} / d r} .
$$

Eqs. (11) and (2) determine the eigenfrequency $\omega$ for the modes, when complemented by proper boundary conditions:

$$
r^{2} \xi_{r}=\frac{l}{\omega^{2}} \frac{\delta p}{\rho_{0}} r \sim r^{l+1} \quad(r \rightarrow 0)
$$

at the stellar center, and

$$
\Delta p=\delta p-\rho_{0} g \xi_{r}=0 \quad(r=R)
$$

for a vanishing Lagrangian perturbation of pressure at the stellar surface $r=R$.

The equilibrium configurations of newly born NSs and SQSs are determined by solving the Newtonian hydrostatic equations, which are consistent with Eqs. (1) and (2). The sound speeds $c_{s}$ and $c_{e}$, thus the Brunt-Väisälä frequency $\omega_{B V}$, are calculated as functions of the radius $r$. Eqs. (11) and (2) are numerically integrated from a small value of $r$ approaching to zero to the stellar surface $r=R$. The outer boundary condition of Eq. (6) is enforced by a shooting routine. The eigenfrequency $\omega$ is computed iteratively after a trial value. The order $n$ of a mode counts the number of nodes in the radial displacement $\xi_{r}$.

To proceed, a NS is assumed to consist of nucleons, electrons, thermal photons and neutrinos trapped in the dense matter, in the first tens of seconds after the core bounce in a core-collapse supernova [25]. A fluid element inside the star is characterized by the baryon density $\rho_{\mathrm{B}}$, the entropy per baryon $S$, and the lepton fraction $Y_{\mathrm{L}}=Y_{\mathrm{e}}+Y_{\nu_{\mathrm{e}}}\left(\right.$ or $Y_{e}$ 
related with $Y_{\mathrm{L}}$ through beta equilibrium conditions, here $\left.Y_{\mathrm{i}}=\rho_{\mathrm{i}} / \rho_{\mathrm{B}}\right)$, all depending on the radial coordinate $r$. In this work all the components except the nucleons are assumed to be non-interacting, while the interactions between nucleons are described by the relativistic mean field (RMF) theory at finite temperature (see, for example, Refs. [26, 27]). We take the formalism of the Lagrangian density for RMF as the same as that used in Ref. [28]. Five parameters for the theory are fixed by fitting the properties of the symmetric nuclear matter at saturation density: saturation nucleon number density $\rho_{0}=0.16 \mathrm{fm}^{-3}$, binding energy $E / A=-16 \mathrm{MeV}$, nucleon effective mass $m^{*}=0.75 m_{N}$ (the bare mass $m_{N}=938 \mathrm{MeV}$ ), incompressibility $K=240 \mathrm{MeV}$ and the symmetry energy $E_{s}=30.5 \mathrm{MeV}$.

Within the framework of RMF theory, we can obtain the energy density and the pressure of the hot NS matter. In the absence of neutrino diffusion, the adiabatic sound speed reads

$$
c_{s}^{2}=\left(\frac{\partial p}{\partial \rho}\right)_{S, Y_{\mathrm{L}}} .
$$

It is remarkable that, in the interior of a newly born NS, the temperature is of order $10 \mathrm{MeV}$ and the timescale of establishing the beta equilibrium can be smaller than $\sim 10^{-8} \mathrm{~S}$ [28], which is much smaller than the period of the $g$-mode pulsations about $10^{-3} \sim 10^{-2} \mathrm{~s}$. Therefore, in our calculation we assume that $Y_{\mathrm{L}}$ for each fluid element is constant and the system is in beta equilibrium during the process of pulsations, which is consistent with the Ledoux convective criterion in supernova simulations [29]. This situation is different from that in cold NS where beta equilibrium can not be obtained during the pulsations and the electron fraction $Y_{\mathrm{e}}$ rather than $Y_{\mathrm{L}}$ remains unchanged [22]. The equilibrium sound speed $c_{e}$ can also be fixed for an equilibrium configuration, where $\rho_{\mathrm{B}}, S$, and $Y_{\mathrm{L}}\left(Y_{e}\right)$ are simply functions of the radius $r$.

For a newly born SQS, it is usually believed to be composed of three flavors of quarks $(\mathrm{u}, \mathrm{d}$ and $\mathrm{s}$ ), electrons, three flavor neutrinos, thermal photons, and gluons. In our present calculation, along the line of Ref. [30], we implement the MIT bag model [31] for its EOS. We choose $m_{\mathrm{u}}=m_{\mathrm{d}}=0, m_{\mathrm{s}}=150 \mathrm{MeV}$, and a bag constant $B^{1 / 4}=154.5 \mathrm{MeV}$, yielding a binding energy per baryon of $928 \mathrm{MeV}$ for the SQS matter in equilibrium $(p=0)$, which is comparable to $931 \mathrm{MeV}$ per nucleon for the ${ }^{56} \mathrm{Fe}$ nucleus [30]. Like in the NS case, all the thermodynamical properties and the two sound speeds can be determined as the equilibrium configuration of a SQS is known.

In our calculation we employ the equilibrium configuration of newly born NSs resulting from the 2D hydrodynamic simulations of core-collapse supernovae by the Arizona Group [32]. We calculate then the properties of the $g$-mode oscillations of newly born NSs at the time $t=100 \mathrm{~ms}, 200 \mathrm{~ms}$ and $300 \mathrm{~ms}$ after the core bounce, along the line taken in Ref. [32]. The time range is limited to within $300 \mathrm{~ms}$ for simplicity since this is guaranteed by the fact that during the first second after the core bounce, the eigenfrequencies vary only 
a little, for example, the frequencies of $g$-modes vary within $727 \mathrm{~Hz} \sim 819 \mathrm{~Hz}$ when the time ranges from $0.3 \mathrm{~s}$ to $1 \mathrm{~s}$ in a newly born NS model [33].

Eqs. (11) and (2) are integrated from the center to a radius of about $20 \mathrm{~km}$ where a convective instable region appears. We choose the mass inside the radius of $20 \mathrm{~km}$ to be $0.8 \mathrm{M}_{\odot}$, $0.95 \mathrm{M}_{\odot}$ and $1.05 \mathrm{M}_{\odot}$ at $t=100 \mathrm{~ms}, 200 \mathrm{~ms}$ and $300 \mathrm{~ms}$ after the core bounce, respectively, according to the supernovae simulations [32]. The increase of the mass with time is due to accretions of mantle materials of the progenitor star onto the newly born neutron star.

Table 1 lists the calculated $g$-mode oscillation eigenfrequencies of an above-modeled newly born NS with order $n=1,2,3$. Here the harmonic index is fixed at $l=2$, which represents the quadrupole motion of mass and is directly related to the emission of gravitational waves (see, for example, Refs. [34, 35, 36]). Our results of $717.6 \mathrm{~Hz}, 774.6 \mathrm{~Hz}$, and $780.3 \mathrm{~Hz}$ for $n=1$ at $t=100 \mathrm{~ms}, 200 \mathrm{~ms}$, and $300 \mathrm{~ms}$ after the core bounce, respectively, are consistent with the Newtonian supernovae simulation results of about $675 \mathrm{~Hz}$ in Ref. [17] and about $950 \mathrm{~Hz}$ in Ref. [20]. They are also close to the general relativistic results ranging from $\sim 620 \mathrm{~Hz}$ to $\sim 820 \mathrm{~Hz}$ in the first second after the core bounce [33]. The quantitative differences result from different equations of state and NS models used, general relativistic effects and so on.

Table 1: Calculated eigenfrequencies (in unit of $\mathrm{Hz}$ ) of the $g$-mode oscillations of the newly born NSs and SQSs at several time $t$ (in unit of millisecond) after the core bounce.

\begin{tabular}{|c|c|c|c|c|c|c|}
\hline \hline \multirow{2}{*}{$\begin{array}{c}\text { Radial order } \\
\text { of } g \text {-mode }\end{array}$} & \multicolumn{3}{|c|}{ Neutron Star } & \multicolumn{3}{c|}{ Strange Quark Star } \\
\cline { 2 - 7 } & $t=100$ & $t=200$ & $t=300$ & $t=100$ & $t=200$ & $t=300$ \\
\hline$n=1$ & 717.6 & 774.6 & 780.3 & 82.3 & 78.0 & 63.1 \\
\hline$n=2$ & 443.5 & 467.3 & 464.2 & 52.6 & 45.5 & 40.0 \\
\hline$n=3$ & 323.8 & 339.0 & 337.5 & 35.3 & 30.8 & 27.8 \\
\hline \hline
\end{tabular}

For comparison, we proceed to calculate the $g$-mode oscillation eigenfrequencies of newly born SQSs. Despite the lack of commonly accepted simulations on supernovae with strange quark cores in literatures, following the results of the trial simulations [37, 38], general theoretical investigations [39, 40] and analysis of some supernovae observations [41, 42] we assume that a strange quark core can be formed during the collapse of presupernova.

To carry out the calculation for SQSs, we assume that the radial variation behaviors of $S$ and $Y_{\mathrm{L}}$ for newly born SQSs are the same as for NSs. But a newly born SQS differs from a NS in its smaller size, having a radius of about $10 \mathrm{~km}$ where the pressure vanishes. With the same masses as a NS at the three time instants after the core bounce, we calculate the $l=2$ eigenfrequencies of a newly born SQS at first. The obtained results are also listed in Table 1, It is apparent that the $g$-mode oscillation frequencies of $82.3 \mathrm{~Hz}, 78.0 \mathrm{~Hz}$, and $63.1 \mathrm{~Hz}$ of a SQS are definitely lower than those of a NS by an order of magnitude. Table 1 also shows the 


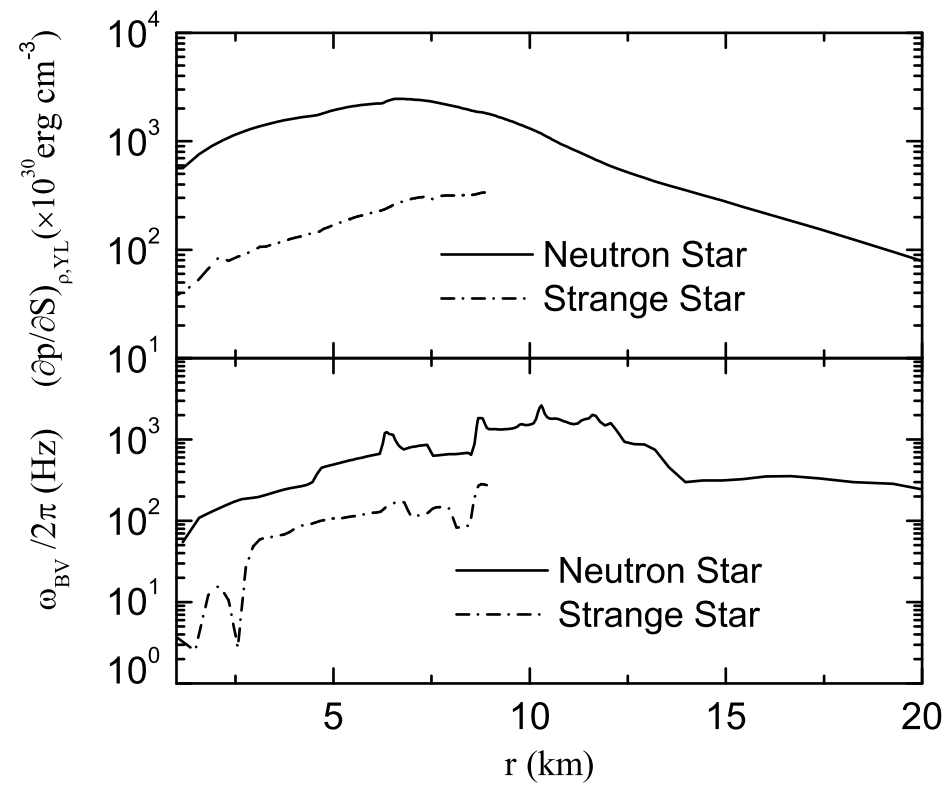

Figure 1: Calculated $(\partial p / \partial S)_{\rho, Y_{L}}$ (top) and Brunt-Väisälä frequency $\omega_{B V}$ (bottom) as functions of the radial coordinate at $t=200 \mathrm{~ms}$ after the core bounce for newly born NSs and SQSs. A sharp surface appears at about $r=9 \mathrm{~km}$ for the SQS.

frequencies of modes with radial order higher than one, although such higher-order $g$-mode oscillations are less likely to be significant sources of gravitational waves, due to the much longer gravitational wave damping time and thus much weaker coupling to gravitational waves [33]. We have also performed calculations in the case of that the entropy per baryon $S$ is increased to $1.5 S$ at each fluid element inside the SQS, by considering the release of thermal energy during the conversion from nuclear matter to strange quark matter [43]. The eigenfrequencies of the $n=1 \mathrm{~g}$-mode oscillations become $100.7 \mathrm{~Hz}, 105.9 \mathrm{~Hz}$, and $96.1 \mathrm{~Hz}$ at the same three time instants, respectively, which are still significantly lower than those of a newly born NS. The dependence of the $g$-mode frequencies on the mass of a SQS has also been investigated. Our finding is that the eigenfrequencies of a newly born SQS increase a little for a larger mass. For example, a SQS core with mass $1.4 \mathrm{M}_{\odot}$ holds the $n=1$ order $g$-mode frequencies $100.2 \mathrm{~Hz}, 91.4 \mathrm{~Hz}$, and $73.0 \mathrm{~Hz}$ at the three instants, respectively. And the frequencies change to $108.8 \mathrm{~Hz}, 100.9 \mathrm{~Hz}$, and $84.5 \mathrm{~Hz}$ accordingly as the mass of the SQS is increased to $1.68 \mathrm{M}_{\odot}$. They are also much lower than those of newly born NSs.

To explore the reason for the large difference in the $g$-mode oscillation eigenfrequencies between newly born NSs and SQSs, we reexpress the Brunt-Väisälä frequency in Eq. (3) as

$$
\omega_{B V}^{2}=-\frac{g}{\rho}\left[\left(\frac{\partial \rho}{\partial Y_{L}}\right)_{p, S} \frac{d Y_{L}}{d r}+\left(\frac{\partial \rho}{\partial S}\right)_{p, Y_{L}} \frac{d S}{d r}\right],
$$


where we further have

$$
\begin{aligned}
& \left(\frac{\partial \rho}{\partial Y_{L}}\right)_{p, S}=-\frac{1}{c_{s}^{2}}\left(\frac{\partial p}{\partial Y_{L}}\right)_{\rho, S}, \\
& \left(\frac{\partial \rho}{\partial S}\right)_{p, Y_{L}}=-\frac{1}{c_{s}^{2}}\left(\frac{\partial p}{\partial S}\right)_{\rho, Y_{L}} .
\end{aligned}
$$

Comparing the calculations for SQSs with those for NSs, we find that the values of the $(\partial p / \partial S)_{\rho, Y_{L}}$ in Eq. (10) for SQSs are much smaller than those for NSs, which result in the values of the Brunt-Väisälä frequency $\omega_{B V}$ for SQSs much smaller than those for NSs as well. Quantitative comparisons of $(\partial p / \partial S)_{\rho, Y_{L}}$ and $\omega_{B V}$ between SQSs and NSs are displayed in Fig. 1 for an illustrative time $t=200 \mathrm{~ms}$. Those calculated results can be understood easily as follows: a SQS is composed of particles which are all extremely relativistic. In the ideal case of neglecting the mass of the strange quark, i.e. $m_{s}=0$, the EOS of a SQS has a simple form as $p=\rho / 3-4 B / 3$ in the MIT model. Substituting this EOS into Eqs. (9) and (10) we find both $(\partial p / \partial S)_{\rho, Y_{L}}$ and $\left(\partial p / \partial Y_{L}\right)_{\rho, S}$ are vanishing and thus the $g$-mode frequency of the SQS in this extreme situation is zero. This simple analysis is confirmed in our numerical calculations when setting $m_{s}=0$. Therefore, the nonvanishing eigenfrequencies of $g$-mode pulsations for a realistic SQS as given in this work are due to the nonzero value of the strange quark mass. It should be emphasized that this result does not depend on the details of the model for the strange quark matter but only on the relativistic properties of the components of SQSs. Once the matter is relativistic and the EOS can be approximately parameterized as $p=\rho / 3+$ const., the $g$-mode frequencies are highly suppressed. While in a NS, beside the relativistic leptons, there are non-relativistic nucleons (or, simply, with quite large masses), which are responsible for the much higher $g$-mode eigenfrequencies.

In summary, we have calculated the eigenfrequencies of $g$-mode pulsations in newly born neutron stars and strange quark stars in this letter. Special emphasis is given to the $g$-mode oscillations with harmonic index $l=2$ and order $n=1$ due to the potential detectability of their gravitational radiation. Because their components are all relativistic particles, the $g$ mode oscillation frequencies of newly born strange quark stars are around or below $100 \mathrm{~Hz}$ for $l=2$ and $n=1$. They are definitely much lower than those of newly born neutron stars $(\sim 650$ - $950 \mathrm{~Hz}$, combining our results and those in literatures) by almost one order of magnitude. Since gravitational waves and the difference of the $g$-mode oscillation frequencies may be detected by LIGO-class detectors, our work illustrates an important signature to distinguish newly born strange quark stars from the neutron stars.

This work was supported by the National Natural Science Foundation of China under contract Nos. 10425521 and 10675007, the Major State Basic Research Development Program under contract No. G2007CB815000, the Key Grant Project of Chinese Ministry of Education under contact No. 305001. 


\section{References}

[1] N. Itoh, Prog. Theor. Phys. 44, 291 (1970) .

[2] B. Freedman, L. McLerran, Phys. Rev. D 17, 1109 (1978).

[3] E. Witten, Phys. Rev. D 30, 272 (1984).

[4] C. Alcock, E. Farhi, and A. V. Olinto, Astrophys. J. 310, 261 (1986).

[5] F. Weber, Prog. Part. Nucl. Phys. 54, 193 (2005).

[6] M. Alford, D. Blaschke, A. Drago, T. Klähn, G. Pagliara, J. Shaffner-Bielich, Nature 445, E 7 (2007).

[7] P. Jaikumar, Eur. Phys. J. C 49, 199 (2007).

[8] F. Özel, Nature 441, 1115 (2006).

[9] F. M. Walter, and J. M. Lattimer, Nature Phys. 2, 443 (2006).

[10] J. M. Lattimer, and M. Prakash, Phys. Rep. 442, 109 (2007).

[11] D. J. Nice et al., Astrophys. J. 634, 1242 (2005).

[12] S. M. Ransom et al., Science 307, 892 (2005).

[13] Q. D. Wang, and T. Lu, Phys. Lett. B 148, 211 (1984).

[14] P. Haensel, J. L. Zdunik, and R. Schaeffer, Astron. Astrophys. 217, 137 (1989).

[15] J. Madsen, Phys. Rev. D 46, 3290 (1992).

[16] C. Schaab, B. Hermann, F. Weber, and M. K. Weigel, Astrophys. J. 480, L111 (1997).

[17] A. Burrows, E. Livne, L. Dessart, C. D. Ott, and J. Murphy, Astrophys. J. 640, 878 (2006); A. Burrows, L. Dessart, C. D. Ott, and E. Livne, Phys. Rep. 442, 23 (2007).

[18] T. Foglizzo, Astron. Astrophys. 368, 311 (2001).

[19] J. M. Blondin, A. Mezzacappa, and C. DeMarino, Astrophys. J. 584, 971 (2003).

[20] C. D. Ott, A. Burrows, L. Dessart, and E. Livne, Phys. Rev. Lett. 96, 201102 (2006).

[21] J. P. Cox, Theory of Stellar Pulsation (Princeton University Press, Princeton, 1980).

[22] A. Reisenegger, and P. Goldreich, Astrophys. J. 395, 240 (1992).

[23] D. Lai, Mon. Not. R. Astron. Soc. 270, 611 (1994).

[24] T. G. Cowling, Mon. Not. R. Astron. Soc. 101, 367 (1941).

[25] H. A. Bethe, Rev. Mod. Phys. 62, 801 (1990).

[26] B. D. Serot, and J. D. Walecka, in Adv. Nucl. Phys., edited by J.W. Negele and E. Voget (Plenum Press, New York, 1986) Vol.16, 1 .

[27] N. K. Glendenning, Compact Stars (Springer-Verlag, Berlin, 2000).

[28] W. J. Fu, G. H. Wang, and Y. X. Liu, Astrophys. J. 678, 1517 (2008). 
[29] R. Buras, M. Rampp, H.-Th. Janka, and K. Kifonidis, Astron. Astrophys. 447, 1049 (2006).

[30] E. Farhi, and R. L. Jaffe, Phys. Rev. D 30, 2379 (1984).

[31] A. Chodos, R. L. Jaffe, K. Johnson, C. B. Thorne, and V. F. Weisskopf, Phys. Rev. D 9, 3471 (1974).

[32] L. Dessart, A. Burrows, E. Livne, and C. D. Ott, Astrophys. J. 645, 534 (2006).

[33] V. Ferrari, G. Miniutti, and J. A. Pons, Mon. Not. R. Astron. Soc. 342, 629 (2003).

[34] C. M. Misner, K. S. Thorne, and J. A. Wheeler, Gravitation (Freeman, New York, 1970).

[35] B. J. Owen, Phys. Rev. Lett. 95, 211101 (2005).

[36] D. Lai, and Y. Q. Wu, Phys. Rev. D 74, 024007 (2006).

[37] L.-M. Lin, K. S. Cheng, M.-C. Chu, and W.-M. Suen, Astrophys. J. 639, 382 (2006).

[38] N. Yasutake, K. Kotake, M.A. Hashimoto, and S. Yamada, Phys. Rev. D 75, 084012 (2007).

[39] P. Jaikumar, S. Reddy, and A. W. Steiner, Phys. Rev. Lett. 96, 041101 (2006).

[40] B. Haskell, N. Andersson, D. I. Jones, and L. Samuelsson, Phys. Rev. Lett. 99, 231101 (2007).

[41] A. B. Chen, T. H. Yu, and R. X. Xu, Astrophys. J. 668, L55 (2007).

[42] D. Leahy, and R. Ouyed, Mon. Not. R. Astron. Soc. 387, 1193 (2008).

[43] J. D. Anand, A. Goyal, V. K. Gupta, and S. Singh, Astrophys. J. 481, 954 (1997). 